\title{
The Environmental Enrichment can Mitigated the Muscular and Memory Adversities Following Stress in Rats
}

\section{Amira Bakeche*, Amina Djouini, Amir Manseur, Mouna Nouacer, Abdelmadjid Bairi, Abdelkrim Tahraoui}

\author{
Applied Neuroendocrinology Laboratory, Department of Biology, Faculty of Sciences, University Badji Mokbtar, \\ Annaba, Algeria.
}

\begin{abstract}
The standard environments commonly used in laboratories obscure the multifaceted parameters of the rodent environment. Thus they can influence their motor skills and their learning. This work proposes to simulate an enriched environment and to study the response to a predation stress according to the nycthemere. We have $32 \mathrm{Wistar}$ rats separated into 08 batches of 04 rats each and placed in two types of accommodation: standard and enriched. At day 01 we have exposed for $5 \mathrm{~min}$ these rats to a predator. At D02, we evaluated its motor coordination by the BWT and between D03 and D07 its cognitive abilities by the Y Maze Test in condition of (16D / 08L as well as 12D / 12L). The results indicate a reduction in stress reactivity within $(12 \mathrm{D} / 12 \mathrm{~L})(04 \pm 0.81)$ wrong step in SE against $(3 \pm 0.81)$ in $\mathrm{EE}$ during dark period, the clear phase is marked by $(0.75 \pm 0.5) \mathrm{SE}$ and $(0.5 \pm 0.57) \mathrm{EE}$. In $(16 \mathrm{D} / 08 \mathrm{~L})$ the same parameter is $(1.5 \pm 0.19)$ in $\mathrm{SE}$ and $(2 \pm 0.81)$ in $\mathrm{EE}$ in clear time, $(1.25 \pm 0.5)$ in $\mathrm{SE}$ and $(1 \pm 0.81)$ in $\mathrm{EE}$ in dark periods. In terms of apprenticeship, EE rats respond better with the correct arm entries in the period (12D / 12L) a change from 1.25 to J03 to 3.75 to J07 in EE vs.1.5 to J03 to 2.75 to J07 in light time SE. In the dark phase it increases from 1.5 to J03 to 3.75 to $\mathrm{J} 07$ in SE and from 2 to J03 to 4.25 to J07 in EE. In (16D / 08L) rats were in a clear period from 0.5 to J03 to 3 to J07 in SE and from 2.5 to J03 to 4 to J07 in EE compared to 1.2 to J03 to 3.25 to J07 in SE and 1.5 to J03 to 3.5 J07 in dark period. As a result, the breeding environment has an interest in the defensive reaction and can impact the behavioural response, even independently of the period of activity.
\end{abstract}

Keywords | Environment, Photoperiod, Stress, Motor skills, Learning

\footnotetext{
Received | October 09, 2019; Accepted | November 14, 2019; Published | December 26, 2019

*Correspondence | Bakeche Amira, Applied Neuroendocrinology Laboratory, Department of Biology, Faculty of Sciences, University Badji Mokhtar, Annaba, Algeria; Email: BakecheAmira@outlook.fr

Citation | Bakeche A, Djouini A, Manseur A, Nouacer M, Bairi A, Tahraoui A (2020). The environmental enrichment can mitigated the muscular and memory adversities following stress in rats. Adv. Anim. Vet. Sci. 8(1): 61-66.

DOI | http://dx.doi.org/10.17582/journal.aavs/2020/8.1.61.66
}

ISSN (Online) | 2307-8316; ISSN (Print) | 2309-3331

Copyright (C) 2020 Bakeche et al. This is an open access article distributed under the Creative Commons Attribution License, which permits unrestricted use, distribution, and reproduction in any medium, provided the original work is properly cited.

\section{INTRODUCTION}

$\mathrm{T}$ he results of clinical and pre-clinical studies have shown that early exposure to environmental and social stressors plays a central role in the development of psychiatric disorders such as depression (Lupien et al., 2009; Pechtel and Pizzagalli, 2011). Many studies focus on physiological approaches to stress management, and the effects of stress management. (Arvin et al., 2017) show that both $\mathrm{CB} 1 \mathrm{R}$ and $\mathrm{CB} 2 \mathrm{R}$ are involved in promoting antidepressant effects in the SIS mouse model. The antidepressant effects of tropisetron $(5 \mathrm{mg} / \mathrm{kg})$ in socially isolated mice are associated with its ability to mitigate the negative effects of iNOS derived $\mathrm{NO}$ on mitochondrial function and redox status in cortical areas of the brain (Arya et al., 2016). Lithium plays a protective role against the convulsive pro-effect mainly mediated by nNOS in the SIS HIPP (Shayan et al., 2016). Other studies address the environmental aspect and how it can alleviate many of the problems of stress reporting the influence of seasons on the frequency of psychiatric hospitalization, suggesting a correlation between seasons and the incidence of mood, stress and depression disorders. such as holiday periods do not seem to influence its seasonal variations, while photoperiod and sunshine intervene more significantly. Improving the confinement conditions of laboratory animals compared to those housed in standard ways refers to environmental enrichment. These conditions 
include larger cages ontaining objects and different spaces to facilitate exercise, play and exploration while allowing animals to better control their environment (Baumans and Van Loo, 2013). Environmental enrichment can enhance learning and information processing (Woodcock and Richardson, 2000). In rodents, predation stress, which is inspired by innate behaviour in the feline repertoire, is one of the most widely used stressors. The aim of this work is to evaluate the impact of environmental enrichment and photoperiod (day = night $/$ day short, long night) on the response to acute phase predation stress in Wistar rats through evaluation of sensor motor functions and memorization.

\section{MATERIAL AND METHODS}

\section{BIOLOGICAL MATERIAL AND BREEDING CONDITIONS}

In the following study, we have prepared 32 white rats under the following species Rattus rattus strain Wistar from the Pasteur Institute of Algiers Algeria. At the beginning of the experiment, the rats were young adults, having $70 \pm 5$ days and weighing on average $135 \mathrm{~g}$. The rats are separated into 08 batches of 4 rats each, following 2 of the 3Rs of the foundation of the ethical approach in animal experimentation: to reduce, refine and replace (Russell and Burch, 1959), from severage to adulthood the rats are subjectedto two types of breeding conditions and are all followed during the day and night time:

02 batches control or standard equinox environment (12 hours day/ 12 hours night)

02 batches controls or standard environment in winter solstice (08 hours day/ 16 hours night)

02 batches housed in enriched environment, in equinox (12 hours day/ 12 hours night)

02 batches housed in enriched environment, in winter solstice (08 hours day/ 16 hours night)

The standard cages are polyethylene, lined with a litter made of wood chips. The enriched cages are screened, lined with cotton and wood chips. These cages were enough large allowing the rats to move freely and facilitates the social interactions between the different congeners physically thanks to the objects at their disposal (plastic toys, wheels, tunnels). Food, in the form of rods based on corn, barley and vitamin supplements and water were both provided ad libitum.

\section{ScheMATIC OF STUDY}

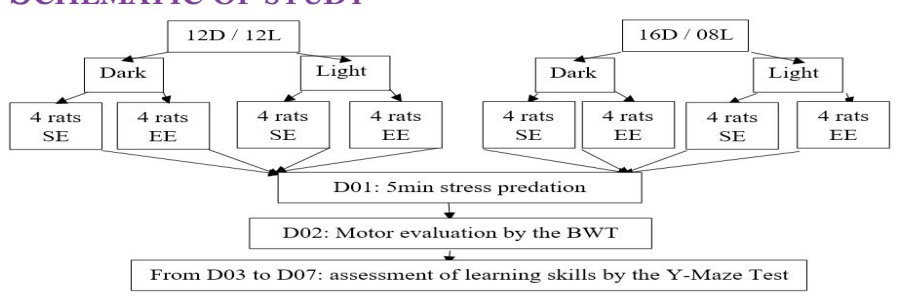

\section{INDUCTION OF STRESS}

After numbering the rats, the predation stress is carried out on day 01. Each session of stress lasts 5 minutes, then the rat is caught in a metal trap inside of a glass arena $(60 \mathrm{~cm} \times 60 \mathrm{~cm})$, with a wooden lid, which encloses a healthy cat. The device allows rats to receive feline stimuli with physical prohibition between predator / prey.

\section{Assessment OF MOTOR ABILITIES}

At the day J02 since the beginning of the experiment, the rats are subjected to the Beam Walking Test. During the BWT, the rats are placed at the end of a bar which measures $1 \mathrm{~m}$ long, $48 \mathrm{~mm}$ wide, and placed $60 \mathrm{~cm}$ above the ground. The video allows the evaluation of the latency to cross the spindle, number of missteps; foot hind leg slides to left or right and number of falls as well (Feeney et al., 1982; Metz et al., 2000).

\section{Evaluation of MEMORY CAPACITIES}

From day 03 to day 07 since the beginning of the experiment, the learning abilities of the rats have been quantified by the Y-Maze test. The device features three $50 \mathrm{~cm}$ long arms, $10 \mathrm{~cm}$ wide and $30 \mathrm{~cm}$ high with an angle of 120 degrees that separates them. It involves observing the subject's ability to remember the arm already visited and the idea that rodents tend to use an optimal search strategy to find a familiar environment in a brand-new situation. Each test does not last for more than 2 minutes and the procedure is repeated for each of the rats 10 times a day for 05 days. The test begins with the deposition at the end of the arms opposite to the starting arms of the bedding of the nest (congener cage). The subject is allowed to choose one of the two arms and stay there for 30 seconds. On the next test, the litter of the previously visited arm is replaced by clean bedding and the unvisited arm becomes the right choice. If it makes the right choice, the subject is allowed to stay 30 seconds and if it chooses the wrong arm, then it is allowed to see that the litter is clean and then it is removed from the labyrinth. The data obtained consists of the number of correct arm entries and the number of incorrect arm entries (Tolman, 1932).

\section{Statistical AnAlysis}

The statistical analysis of the data was carried out using SPSS version 25.0 (SPSS, 2017). The results were represented in the form of mean \pm standard deviation of mean $(\mathrm{M} \pm \mathrm{SEM})$ and compared by a test of student in significance threshold Alpha $=0.05$.

\section{RESULTS AND INTERPRETATIONS}

In EE rats the latency before starting the test in $12 \mathrm{~L} / 12 \mathrm{D}$ condition is $(3 \pm 1.41) \mathrm{S}$ in light phase and $(30 \pm 15) \mathrm{S}$ in dark phase. This time is not significantly different from that of SE rats: $(6.5 \pm 2.79) \mathrm{S}$ in light phase and $(49.75 \pm$ 
13.13) S in dark phase. In condition 08L / 16D the latency before starting the test is not significantly higher in EE rats: $(24.25 \pm 10.81) \mathrm{S}$ in light phase and $(18.5 \pm 5.51) \mathrm{S}$ in dark phase vs. only in SE rats: $(16 \pm 7.48) \mathrm{S}$ in light phase and $(11.25 \pm 3.06) \mathrm{S}$ in dark phase (Figure 1).

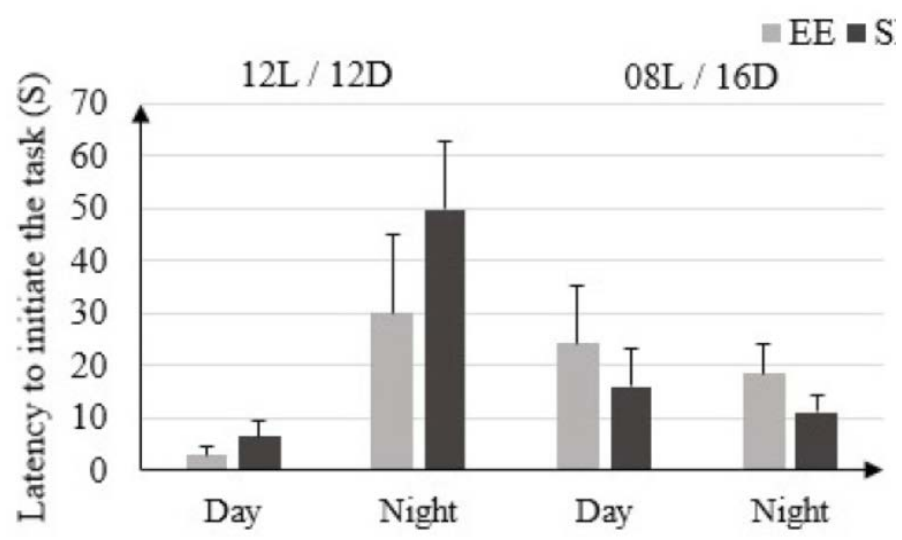

Figure 1: Latency to initiate the task (Seconds) in rats housed in enriched environment (EE) and rats housed in standard environment (SE) under 12L / 12D and 08L / $16 \mathrm{D}$ conditions in the Beam Walking Test.

In the 12L/ 12D condition, the EE and SE rats reported a significantly higher false step (right and left) in light phase with respectively $(3 \pm 0.81)$ and $(4 \pm 0.81)$ than in dark phase $(0.5 \pm 0.57)$ and $(0.75 \pm 0.5)$. In $08 \mathrm{~L} / 16 \mathrm{D}$ this same parameter shows no significant difference between $\mathrm{EE}$ and SE rats in both phases: $(2 \pm 0.81)$ EE light, $(1.5 \pm 0.19)$ SE light and $(1 \pm 0.81)$ EE dark, $(1.25 \pm 0.5)$ SE dark. The number of falls during the trial is 0 in both types of environments, during both phases in 12L / 12D condition. In condition $08 \mathrm{~L} / 16 \mathrm{D}$ the numbers of falls during the trial is 0 in EE rats during both phases and $(0,5 \pm 0.57)$ in SE rats in light phase and $(0.25 \pm 0.5)$ in dark phase (Figure 2).

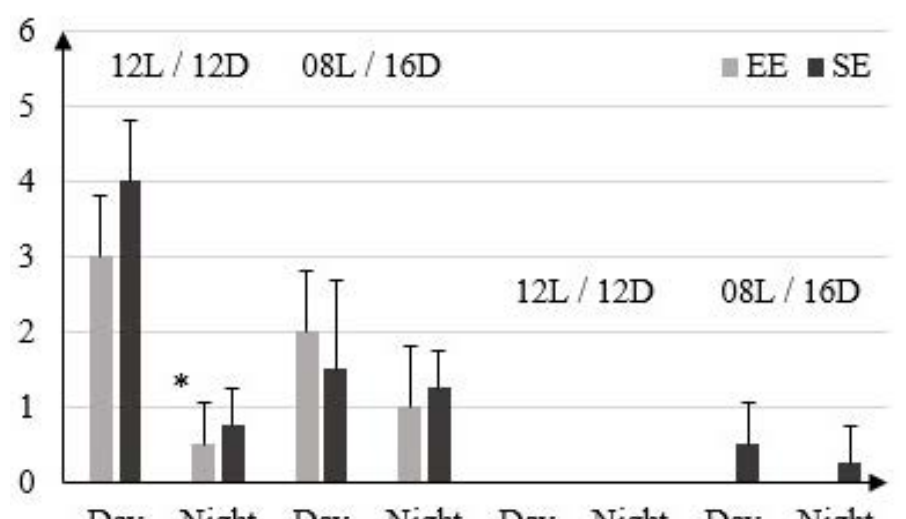

Day Night Day Night Day Night Day Night

Hing leg foot slips (right and left) Number of falls during the trial

Figure 2: Number of false step (right and left) and number of falls during the trial in rats housed in enriched environment (EE) and rats housed in standard environment (SE) in 12L / 12D and 08L / 16D in Beam Walking Test.
In $12 \mathrm{~L} / 12 \mathrm{D}$, there is no significant difference in the light phase between the number of correct arm entries in the rats housed in EE and those in the SE, which increases from 1.25 to $\mathrm{D} 1$ to 3.75 to $\mathrm{D} 5$ for the EE rats and from 1.5 to D1 to 2.75 to D5 for the rats of the SE (Figure 3).

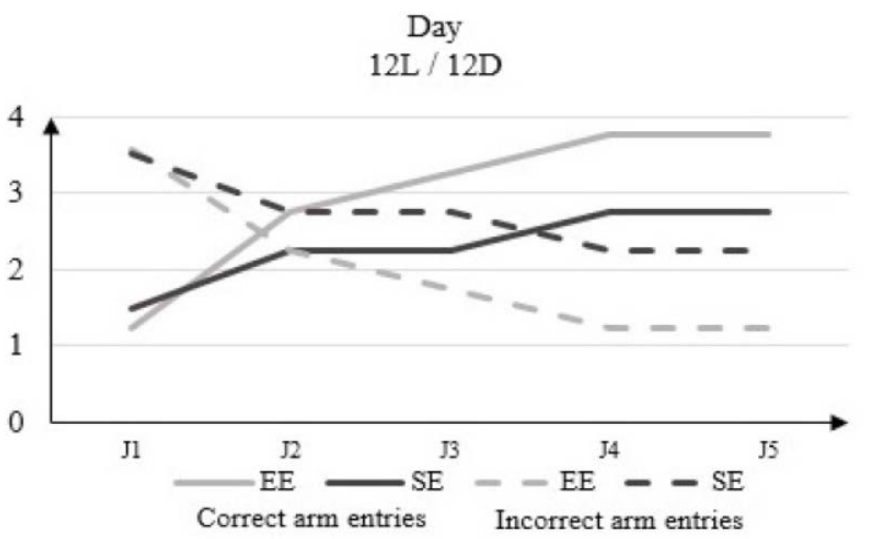

Figure 3: Correct arm entries and incorrect arm entries numbers in rats housed in enriched environment (EE) and rats housed in standard environment (SE) in clear phase $(12 \mathrm{~L} / 12 \mathrm{D})$ in the Y-Maze Test.

In light phase conditions of $08 \mathrm{~L} / 16 \mathrm{D}$ there is a significant difference in the number of correct arm entries between EE rats that range from 2.5 to D1 to 4 to D5 and those of the SE of 0.5 to D1 to 3 to D5 (Figure 4).
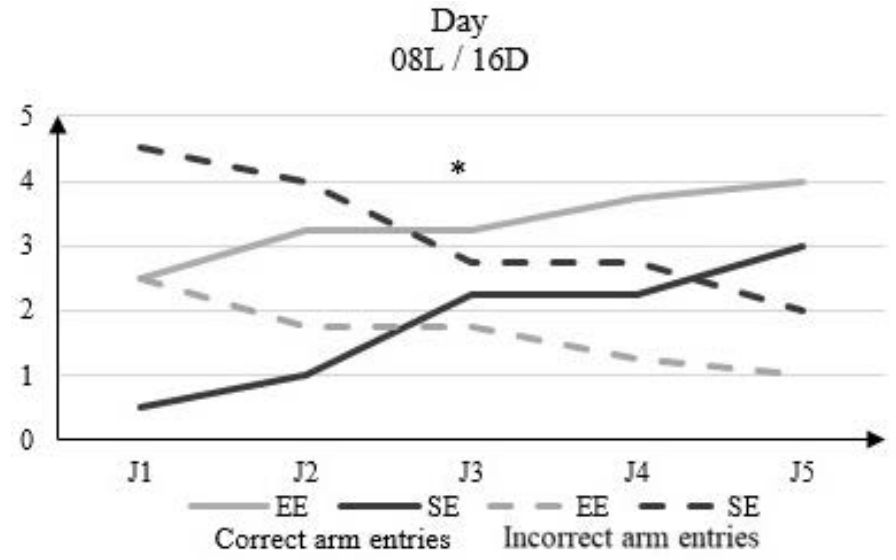

Figure 4: Correct arm entries and incorrect arm entries numbers in rats housed in enriched environment (EE) and rats housed in standard environment (SE) in clear phase (08L / 16D) in the Y-Maze Test.

In the dark phase of $12 \mathrm{~L} / 12 \mathrm{D}$, the correct arm entries number changes from 2 to D1 to 4.25 to D5 in the rats housed in EE and from 1.5 to D1 to 3.75 to D5 in rats housed in SE (Figure 5).

In the dark phase of the 08L / 16D, the correct arm entries number in the rats housed in EE increased from 1.5 to D1 from the beginning of the test to 3.5 to D5. In rats housed in $\mathrm{SE}$ this number goes from 1.2 to D1 from the beginning 
of the test to 3.25 to D5 (Figure 6).

Night

$12 \mathrm{~L} / 12 \mathrm{D}$

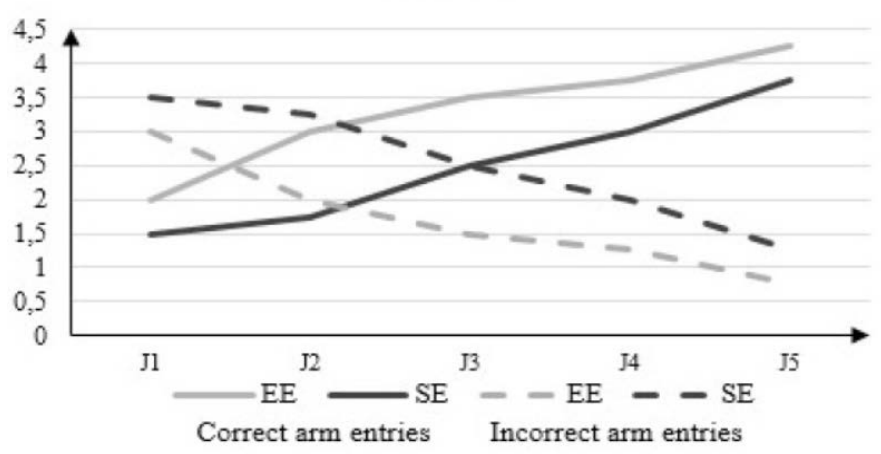

Figure 5: Correct arm entries and incorrect arm entries numbers in rats housed in an enriched environment (EE) and rats housed in a standard environment (SE) in the dark phase (12L / 12D) in the Y-Maze Test.

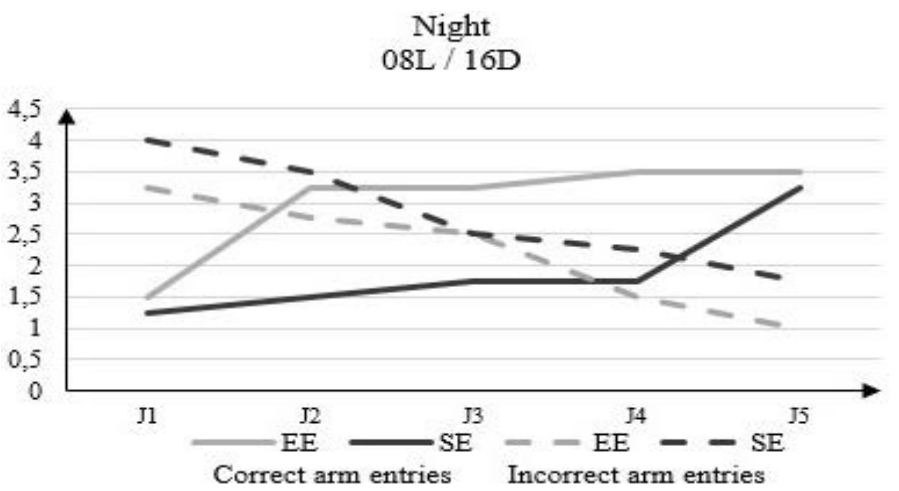

Figure 6: Correct arm entries and incorrect arm entries numbers in rats housed in an enriched environment (EE) and rats housed in standard environment (SE) in the dark phase (08L / 16D) in the Y-Maze Test.

\section{DISCUSSION}

In an acute condition, stress modulates the behavioural response so that it is adapted to the situation. Thus, it allows to modulate different higher functions such as learning and memorisation (Schwabe et al., 2010a) In fact this even explain that the stress could indeed shape the memory, they give details to these remarks by demonstrating that the stress plays a major role in the quantity and quality of memory. Regarding the quantitative aspect, it dependents on both the context and the emotional state of the subject. Some authors report stress-facilitating effects on the acquisition, consolidation and recall of new information (Nater et al., 2007; Schwabe et al., 2008) others on the other hand on disruptive effects (Lupien et al., 1997). Amy and Arnsten, 2009, Schwabe and Wolf, 2010). There are several factors to consider such as the emotional context in which the learning is done, the complexity of the task and the intensity of the stimulus. Overall, the observed performances depend on these factors, and thus vary with the intensity of stress (Diamond et al., 1992; Diamond et al., 2007).

Concerning the qualitative aspect, the stress will modulate the behavioural response increasing by this way the establishment of the stimulus-response associations, depending on the striatum, or of stimulus-stimulus (spatial) type of associations, depending on the Hippocampus (Schwabe et al., 2010b). Stress therefore forces the subject to employ simple, fast and erect strategies to the detriment of flexible cognitive strategies that are adaptable to new situations.

In 1947, Donald Hebb discovered that rats raised near humans, much like pets, solved labyrinth problems better than those raised in the individual cage laboratory. Hebb was the first to develop the concept of enriched environment, in particular by describing the free environment, a condition in which rats can move freely in a large room. He argued that experience and enrichment of the environment would influence the neuronal organization of the brain (Hebb, 1949).

Our results support the above references and show that in the Y-Maze test, the post-traumatic cognitive performances of the rats housed in enriched medium are all more significant comparing to those of the depleted or standard medium during the two phases of the nycthemere During the period $(12 \mathrm{D} / 12 \mathrm{~L})$ there was a change in terms of correct arm entries from 1.25 to D03 to 3.75 to D07 in EE compared to 1.5 to D03 to 2.75 to D07 in SE in clear time. In the dark phase it increases from 1.5 to D03 to 3.75 to D07 in SE and from 2 to D03 to 4.25 to D07 in EE. In (16D / 08L) rats were in a clear period from 0.5 to D03 to 3 to D07 in SE and from 2.5 to D03 to 4 to D07 in EE compared to 1.2 to D03 to 3.25 to D07 in SE and 1.5 to D03 to 3.5 D07 in dark period. Environmental enrichment maintains both a high learning and enhance memorization skills in aversive conditions even outside the activity phase.

Spontaneous locomotors activity can be used as a simple index of information processing or learning that reflects the body's ability to adapt effectively to its environment (Elliott and Grunberg, 2005).

Rats housed in an enriched environment adapt and explore the new environment better than those left under standardized conditions. At BWT, for example, the latency time before starting the $(12 \mathrm{D} / 12 \mathrm{~L})$ stain is $(6.5 \pm 2.79) \mathrm{S}$ in $\mathrm{SE}$ and $(3 \pm 1.41) \mathrm{S}$ in $\mathrm{EE}$ in clear time versus $(49.75 \pm 13.13)$ in $\mathrm{SE}$ and $(30 \pm 15) \mathrm{S}$ in obscure time seal. Environmental enrichment has improved sensor motor performance in the Beam Walking Test (Christie and Dalrymple-Alford, 1995). 
The beneficial effects of environmental enrichment on motor coordination from the Beam Walking Test in our study are asserted. Rats housed in this type of environment perform more tactile tasks. with $(12 \mathrm{D} / 12 \mathrm{~L})(04 \pm 0.81)$ wrong step in $\mathrm{SE}$ against $(3 \pm 0.81)$ in $\mathrm{EE}$ in dark period, the clear phase is marked by $(0.75 \pm 0.5)$ in SE and $(0.5 \pm 0.57)$ in EE. In $(16 \mathrm{D} / 08 \mathrm{~L})$ the same parameter is $(1.5 \pm 0.19)$ in $\mathrm{SE}$ and $(2 \pm 0.81)$ in EE in clear time, $(1.25 \pm 0.5)$ in $\mathrm{SE}$, and $(1 \pm 0.81)$ in $\mathrm{EE}$ in dark timexEnvironmental enrichment appears to exacerbate the motor faculties regardless of the photoperiod.

\section{CONCLUSION}

This work demonstrates how environmental enrichment impacts the adaptive response to aversive stress regardless of sunshine. This type of environment has the ability to synchronize the activity as a function of the day by damping the physiological imbalance and improving its defensive response.

\section{AUTHORS CONTRIBUTION}

All authors contributed equally.

\section{CONFLICT OF INTEREST}

The authors declare that there is no conflict of interest.

\section{REFERENCES}

- Amy F, Arnsten AF (2009). Stress signaling pathways that impair prefrontal cortex structure and function. Nat. Rev. Neurosci. 10(6): 410 - 422.https://doi.org/10.1038/nrn2648

-Arvin HM, Hossein AK, Arya H, Shayan A, Maria G, Mazyar Z, Hamed S, Ahmad RD (2017). Activation of cannabinoid receptors elicits antidepressant-like effects in a mouse model of social isolation stress. Brain. Res. Bull. 130: 200 - 210. https://doi.org/10.1016/j.brainresbull.2017.01.018

-Arya HM, Shayan A, Hossein AK, Maryam RB, Nastaran K, Carl OO, Mojgan R, Parvaneh N, Hassan M, Ahmad R, Mir-Jamal H, Elika S, Shahram EM (2016). Attenuation of oxidative and nitrosative stress in cortical area associates with antidepressant-like effects of tropisetron in male mice following social isolation stress. Brain. Res. Bull. 124: 150 163. https://doi.org/10.1016/j.brainresbull.2016.04.018

-Baumans V, Van Loo PL (2013). How to improve housing conditions of laboratory animals: the possibilities of environmental refinement. Vet. J. 195(1): 24 - 32. https:// doi.org/10.1016/j.tvj1.2012.09.023

- Christie MA, Dalrymplealford JC (1995). Behavioral consequences of frontal cortex grafts and enriched environments after sensorimotor cortex lesions. J. Neural. Transp. Plast. 5(4): 199-210. https://doi.org/10.1155/ NP.1994.199

- Cohen H, Kozlovsky N, Alona C, Matar MA, Joseph Z (2012). Animal model for PTSD: from clinical concept to translational research. Neuropharmacology, 62(2): 715 -724.

https://doi.org/10.1016/j.neuropharm.2011.04.023

-Diamond DM, Bennett MC, Fleshner M, Rose GM (1992). Inverted- $U$ relationship between the level of peripheral corticosterone and the magnitude of hippocampal primed burst potentiation. Hippocampus. 2(4): 421- 430. https:// doi.org/10.1002/hipo.450020409

- Diamond DM, Campbell AM, Park CR, Halonen J, Zoladz PR (2007). The temporal dynamics model of emotional memory processing: a synthesis on the neurobiological basis of stressinduced amnesia, flashbulb and traumatic memories, and the Yerkes-Dodson law. Neural. Plast. 2007: 60803. https://doi. org/10.1155/2007/60803

-Elliott BM, Grunberg NE (2005). Effects of social and physical enrichment on open field activity differ in male and female Sprague-Dawley rats. Behav. Brain. Res. 165(2): 187 - 196. https://doi.org/10.1016/j.bbr.2005.06.025

- Feeney DM, Gonzalez A, Law WA (1982). Amphetamine, haloperidol, and experience interact to affect rate of recovery after motor cortex injury. Science. 217(4562) : 855 - 857. https://doi.org/10.1126/science.7100929

- Hebb D (1949). The organization of behavior. In: A neuropsychological theory, New York, Wiley, 6: 307-307.

-Lupien SJ, Gaudreau S, Tchiteya BM, Maheu F, Sharma S, Nair NP, Hauger RL, Mcewen BS, Meaney MJ (1997). Stress-induced declarative memory impairment in healthy elderly subjects: relationship to cortisol reactivity. J. Clin. Endocr. Metab. 82(7): 2070-2075. https://doi.org/10.1210/ jcem.82.7.4075

-Lupien SJ, McEwen BS, Gunnar MR, Heim C (2009). Effects of stress throughout the lifespan on the brain, behaviour and cognition. Nat. Rev. Neurosci. 10 (6): 434 - 445. https://doi. org/10.1038/nrn2639

- Metz GA, Merkler D, Dietz V, Schwab ME, Fouad K (2000). Efficient testing of motor function in spinal cord injured rats. Brain. Res. 883(2): 165 - 177. https://doi.org/10.1016/ S0006-8993(00)02778-5

- Nater UM, Moor C, Okere U, Stallkamp R, Martin M, Ehlert U, Kliegel M (2007). Performance on a declarative memory task is better in high than low cortisol responders to psychosocial stress. Psychoneuroendocrino. 32(6): 758 - 763. https://doi.org/10.1016/j.psyneuen.2007.05.006

- Pechtel P, Pizzagalli DA (2011). Effects of early life stress on cognitive and affective function: an integrated review of human literature. Psychopharmacology, 214 (1): 55-70. https://doi.org/10.1007/s00213-010-2009-2

- Russell WMS, Burch RL (1959). The Principles of Humane Experimental Technique. London, UK: Methuen, xiv +: 238.

- Schwabe L, Bohringer A, Chatterjee M, Schachinger H (2008). Effects of pre-learning stress on memory for neutral, positive and negative words: Different roles of cortisol and autonomic arousal. Neurobiol. Learn. Mem. 90(1): 44-53. https://doi.org/10.1016/j.nlm.2008.02.002

- Schwabe L, Schachinger H, de Kloet ER, Oitzl MS (2010b). Stress impairs spatial but not early stimulus-response learning. Behav. Brain. Res. 213(1): 50-55. https://doi. org/10.1016/j.bbr.2010.04.029

-Schwabe L, Wolf OT (2010). Stress impairs the reconsolidation of autobiographical memories. Neurobiol. Learn. Mem. 94(2): 153-157. https://doi.org/10.1016/j.nlm.2010.05.001

- Schwabe L, Wolf OT, Oitzl MS (2010a). Memory formation under stress: quantity and quality. Neurosci. Biobehav. R. 34(4): 584-591. https://doi.org/10.1016/j. 
- Shayan A, Arya HM, Hossein Ak, Armin S, Maryam RB, Ali R, HB, Mojgan R, Nastaran K, Arvin HM, Shahram EM, Ahmad RD (2016). Lithium attenuates the proconvulsant effect of adolescent social isolation stress via involvement of the nitrergic syste. Brain. Res. Bull. 61: 613. https://doi.org/10.1016/j.yebeh.2016.04.035

-SPSS (2017). IBM Corp. IBM SPSS Statistics for Windows,

-Tolman EC (1932). Purposive behavior in animals and men. New York: Appleton-Century.

-Woodcock EA, Richardson R (2000). Effects of multisensory environmental stimulation on contextual conditioning in the developing rat. Neurobiol. Learn. Mem. 74(2): 89-104. https://doi.org/10.1006/nlme.1999.3949 